\title{
Time-resolved local strain tracking microscopy for cell mechanics
}

O. Aydin' , B. Aksoy', O. B. Akalin, H. Bayraktar', and B. E. Alaca'

Citation: Review of Scientific Instruments 87, 023905 (2016); doi: 10.1063/1.4941715

View online: http://dx.doi.org/10.1063/1.4941715

View Table of Contents: http://aip.scitation.org/toc/rsi/87/2

Published by the American Institute of Physics

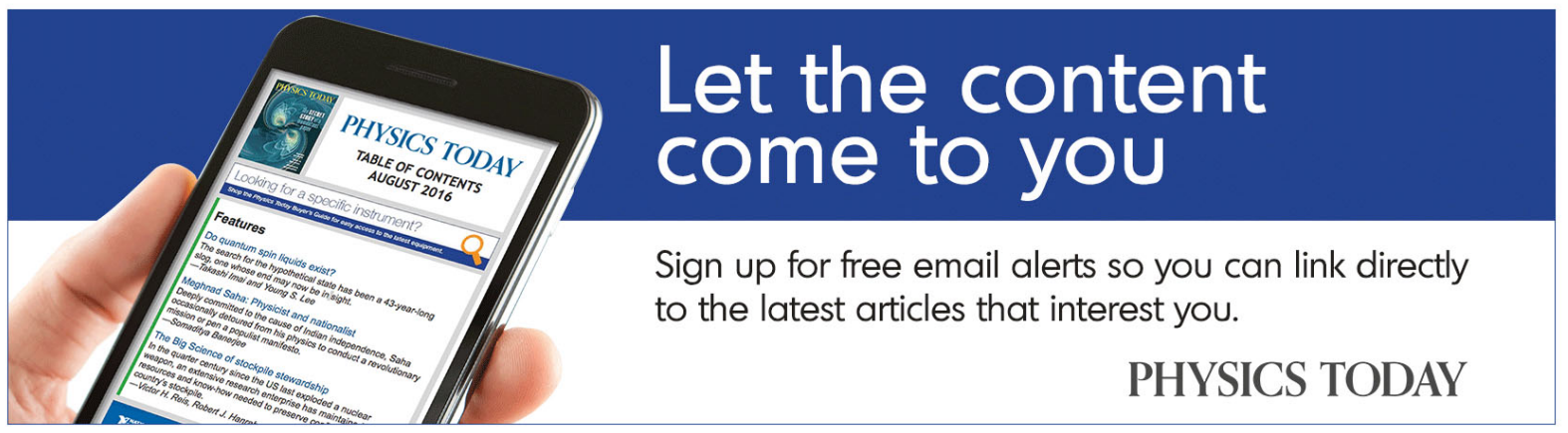




\title{
Time-resolved local strain tracking microscopy for cell mechanics
}

\author{
O. Aydin, ${ }^{1, a)}$ B. Aksoy, ${ }^{1, a)}$ O. B. Akalin, ${ }^{2}$ H. Bayraktar, ${ }^{2,3, b)}$ and B. E. Alaca ${ }^{1,4, b)}$ \\ ${ }^{1}$ Department of Mechanical Engineering, Koç University, Rumelifeneri Yolu, Sariyer 34450 Istanbul, Turkey \\ ${ }^{2}$ Biomedical Science and Engineering, Koç University, Rumelifeneri Yolu, Sariyer 34450 Istanbul, Turkey \\ ${ }^{3}$ Department of Chemistry, Koç University, Rumelifeneri Yolu, Sariyer 34450 Istanbul, Turkey \\ ${ }^{4}$ Koç University Surface Science and Technology Center, Rumelifeneri Yolu, Sariyer 34450 Istanbul, Turkey
}

(Received 7 August 2015; accepted 25 January 2016; published online 12 February 2016)

\begin{abstract}
A uniaxial cell stretching technique to measure time-resolved local substrate strain while simultaneously imaging adherent cells is presented. The experimental setup comprises a uniaxial stretcher platform compatible with inverted microscopy and transparent elastomer samples with embedded fluorescent beads. This integration enables the acquisition of real-time spatiotemporal data, which is then processed using a single-particle tracking algorithm to track the positions of fluorescent beads for the subsequent computation of local strain. The present local strain tracking method is demonstrated using polydimethylsiloxane (PDMS) samples of rectangular and dogbone geometries. The comparison of experimental results and finite element simulations for the two sample geometries illustrates the capability of the present system to accurately quantify local deformation even when the strain distribution is non-uniform over the sample. For a regular dogbone sample, the experimentally obtained value of local strain at the center of the sample is $77 \%$, while the average strain calculated using the applied cross-head displacement is $48 \%$. This observation indicates that considerable errors may arise when cross-head measurement is utilized to estimate strain in the case of non-uniform sample geometry. Finally, the compatibility of the proposed platform with biological samples is tested using a unibody PDMS sample with a well to contain cells and culture media. HeLa S3 cells are plated on collagen-coated samples and cell adhesion and proliferation are observed. Samples with adherent cells are then stretched to demonstrate simultaneous cell imaging and tracking of embedded fluorescent beads. @ 2016 AIP Publishing LLC. [http://dx.doi.org/10.1063/1.4941715]
\end{abstract}

\section{INTRODUCTION}

Studies have emerged in the past few decades investigating the effects of mechanical stimulation on biological cells. It is now the commonly accepted view that cells are subjected to mechanical stress in vivo which is transmitted to the cell through the extracellular matrix activating mechanosensitive pathways and inducing a cellular response. ${ }^{1}$ In the in vitro studies of mechanobiology, cells are subjected to engineered conditions where the mechanical state of the relevant in vivo environment is simulated. A common approach is to plate cells on a flexible membrane, deform the cells indirectly by stretching the membrane, ${ }^{2}$ and use optical microscopy to assess cell response to stretching.

One of the most thoroughly studied phenomena in mechanobiology is the morphological reorientation response of cells to cyclic stretch, which was observed in the earlier cell stretching studies on endothelial cells, ${ }^{3-6}$ smooth muscle cells, ${ }^{7,8}$ and fibroblasts. ${ }^{9}$ The stretch-induced cell reorientation phenomenon has been revisited and studied in greater detail in the past few years utilizing the advances in instrumentation, imaging, and computational technologies. The dependence of cell reorientation response on stretching frequency, ${ }^{10}$ effects of non-uniform strain fields on cell reorientation, ${ }^{11,12}$ concurrent

\footnotetext{
a) O. Aydin and B. Aksoy contributed equally to this work.

b) Authors to whom correspondence should be addressed. Electronic addresses: hbayraktar@ku.edu.tr and ealaca@ku.edu.tr
}

realignment of actin cytoskeleton, ${ }^{13-15}$ and reorganization of focal adhesion sites ${ }^{15-18}$ were investigated.

In addition to the well-documented cell reorientation response, cell-stretching studies have also revealed numerous mechanically induced intracellular responses such as protein synthesis, ${ }^{19}$ DNA synthesis ${ }^{20}$ and phosphorylation of extracellular signal-regulated kinases ${ }^{21-23}$ and mitogen-activated protein kinases. ${ }^{24}$ Furthermore, cell stretching was also utilized to study the effects of stretch on cell traction forces, ${ }^{25}$ wound closing, ${ }^{26}$ cell death, ${ }^{27,28}$ cell migration, ${ }^{29}$ and differentiation. ${ }^{30}$

Cell stretching is a key technique that can be used to study the effects of mechanical stimuli on cell morphology and behavior. However, the simulated in vitro mechanical environment is often poorly characterized. The commonly employed measure of applied mechanical deformation is the percentage stretch, which is calculated as the ratio of elongation or cross-head displacement to original length of the sample, i.e., the cross-head strain. The magnitude of crosshead strain is a one-dimensional scalar value that corresponds to the average longitudinal strain. When a membrane with adherent cells is deformed, cells are subjected to a twodimensional strain field that can be characterized in terms of the longitudinal, transverse, and shear components of strain. Moreover, in-plane strain components may have a non-uniform spatial distribution, if the sample has heterogeneous mechanical properties or non-uniform cross-sectional area. $^{31}$ 
The distribution of strain over the sample is relevant to cell stretching experiments since samples often have non-uniform cross-sectional areas due to wells that contain cells and culture media. When the sample has a non-uniform strain field in a cell stretching experiment, local strain in the vicinity of cells in question may be different from the applied cross-head strain since the latter is an average value. Furthermore, a nonuniform strain field can result in adherent cells being subjected to strain gradients, which is known to affect cell behavior. ${ }^{13}$ Characterization of the two-dimensional strain field is therefore an important requirement for an adequate and accurate description of applied deformation in cell stretching.

Several groups have addressed this issue in the past. A few studies focused on non-uniform strain fields using finite element modeling $(\mathrm{FEM})^{32}$ or experimental measurements ${ }^{12}$ to quantify sample deformation. In most other cases, uniformity of the strain field in the region of the sample where cells are seeded was verified by FEM, ${ }^{33}$ experimental measurements, ${ }^{10,34,35}$ or both. ${ }^{13,15,23,26}$ Although these studies provide detailed analysis of relevant sample deformation, characterization of local strain field and cell imaging are not carried out simultaneously. These previously reported cell-stretching systems are characterized by analyzing the strain field without seeding the cells on the sample, and this is used as a calibration for subsequent cell stretching experiments.

In this article, a cell stretching system capable of cell imaging and concurrent time-resolved measurement of local strain field is presented. The present approach is based on the integration of a uniaxial stretcher device with fluorescent microscopy, using transparent polymer membranes with embedded fluorescent beads, and computation of local strain field from the displacements of these beads. The role of the custom-built tensile stretcher is to deform the sample while fluorescent microscopy images of the beads are recorded during stretching. The trajectories of the fluorescent beads are tracked by implementing image processing on consecutive frames. Local strain is then computed from the displacements of the beads. The major advantage of the present cell stretching system is the ability to measure local strain field and image adherent cells simultaneously, enabling accurate and timeresolved correlation between applied mechanical deformation and cell response.

To demonstrate the capability of the present method to accurately measure local strain, the longitudinal component of the local strain is compared with the applied cross-head strain for two different sample geometries: (1) a rectangular geometry, where strain is expected to be distributed uniformly, when one considers points sufficiently away from the grips, and (2) a dogbone geometry, where much of the deformation is confined to the narrow neck region and the local strain at the neck region is expected to be larger than the applied crosshead strain. ${ }^{36}$ Both samples are prepared using polydimethylsiloxane (PDMS) elastomer. FEM is performed for both sample geometries to predict the strain distribution and validate experimental measurements. To conclude, compatibility of the present system with biological samples as well as cell stretching and simultaneous particle-tracking are demonstrated by plating HeLa S3 cells on PDMS and stretching the samples with adherent cells.

\section{EXPERIMENTAL SETUP AND OPERATION}

\section{A. Design of uniaxial tensile stretcher}

The overall mechanical design of the uniaxial tensile stretcher is laid out with focus on flexibility, easy customization, and most importantly, compatibility with inverted microscope setups. The stretcher is depicted in top and front views in Figs. 1(a) and 1(b), respectively. It utilizes a linear actuator (Part 1) that applies tensile load, and a load cell (Part 10) to measure the tension in the specimen. The linear actuator is a stepper motor with a lead screw (Part 2) as the motor shaft. The sample (Part 9) is gripped tightly at two ends by custom-made aluminum grips (Part 8). One of the grips is movable while the other is fixed. The movable grip is actuated by the stepper motor and a linear guide rail system (Part 4) and carts (Part 5) are used to achieve a stable linear motion of the grip. The movable grip is attached to the motor shaft via a mounting piece (Part 6) and the fixed grip is attached to the load cell via another mounting piece (Part 7). The grip mounting pieces are designed for permanent use, while the grips themselves can easily be replaced. Hence, a variety of different grips can be used with samples of different sizes and shapes. The linear actuator, load cell, and the grips are

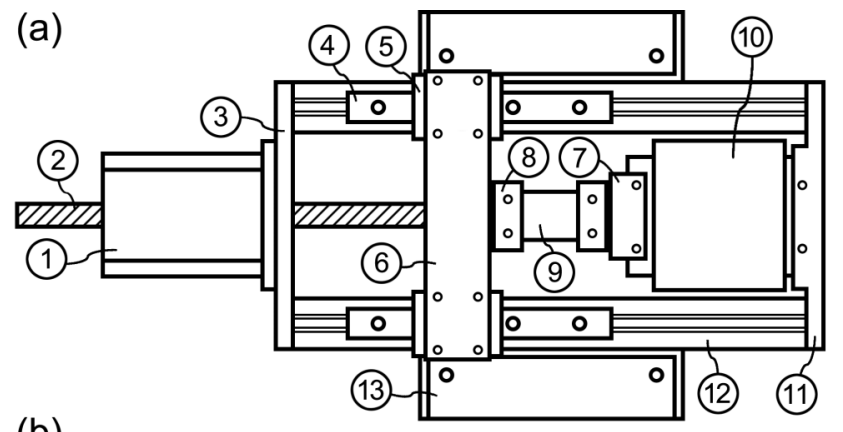

(b)
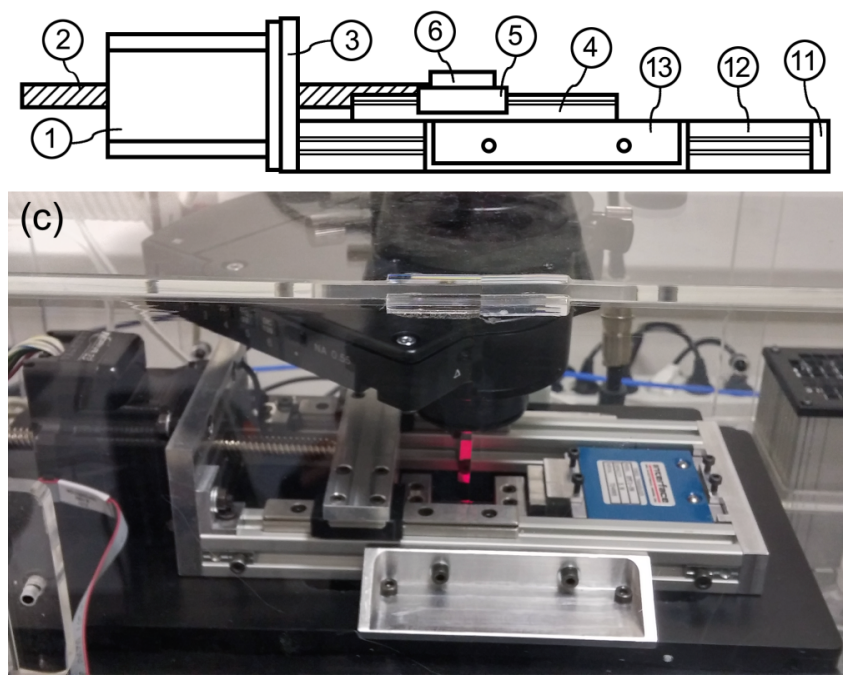

FIG. 1. Schematic (a) top view and (b) front view of the stretcher illustrating the basic components: The stepper motor (1) and lead screw (2), motor mounting plate (3), linear guide rails (4) and carts (5), movable grip mounting piece (6), fixed grip mounting piece (7), grips (8), specimen (9), load cell (10), load cell mounting plate (11), T-slotted aluminum extrusion pieces (12), and brackets for mounting the stretcher onto a microscope stage (13). (c) View of the tensile stretcher mechanism mounted onto the stage of an inverted microscope. 
assembled onto two pieces of T-slotted aluminum extrusion pieces (Part 12) that make up the backbone of the stretcher and enable easy mounting and customization. The entire assembly can be mounted onto most inverted microscope stages using simple L-shaped brackets (Part 13) that can be custom-made for the microscope stage and attached to the T-slotted extrusion pieces on the stretcher assembly. The stretcher-microscope assembly is shown in Fig. 1(c).

\section{B. System characterization}

The uniaxial tensile stretcher is operated and controlled using a PC and the LabVIEW development environment. Both the stepper motor and the load cell are powered by a DC power supply and the appropriate electrical connections are made between the motor, load cell, and the PC. A block diagram of the stretcher system is shown in Fig. 2(a), detailing the electrical connections between the power supply, stepper motor, load cell, and the PC.

The linear stepper actuator (IMS MDrive23 ${ }^{\mathrm{TM}}$ Series) has an integrated driver and a programmable controller with two
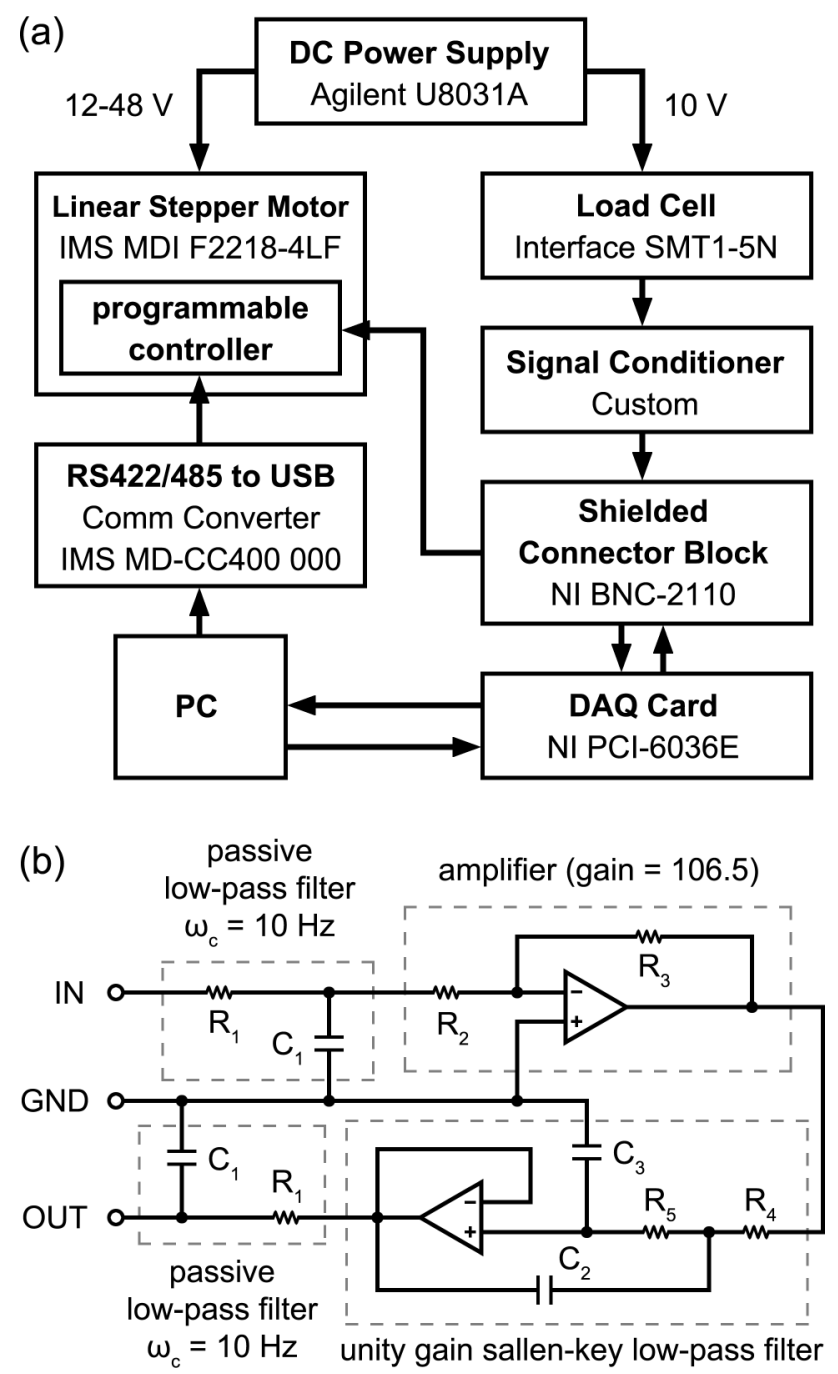

FIG. 2. (a) Block diagram of the stretcher system and (b) schematic of the custom built signal conditioner circuit for filtering and amplifying load cell readout signal. communication interfaces; a general purpose digital $\mathrm{I} / \mathrm{O}$ port and an RS-485 communication port. The RS-485 communication port is connected to the $\mathrm{PC}$ via a communication converter and is used to assign functions to the general-purpose I/O channels for driving the motor. The general-purpose I/O channels are then connected to the $\mathrm{PC}$ via a shielded connector block (NI BNC-2110) and a DAQ (Data Acquisition) card (NI PCI-6036E), and the motor is controlled through this interface using a LabVIEW subroutine.

The tension in the specimen is measured by a load cell (Interface SMT1-5N) consisting of strain gauges configured as a Wheatstone bridge. A DC power supply provides $10 \mathrm{~V}$ excitation to the load cell and the output signal is filtered and amplified using a custom signal conditioner circuit as depicted in Fig. 2(b). The conditioned output is then connected to the PC through the shielded connector block and DAQ card, and the same LabVIEW subroutine that controls the linear stepper motor is used to record the load cell output.

Due to the lack of an encoder or a displacement sensor to provide displacement feedback for actuation, the linear actuator is controlled in an open loop configuration. This requires meticulous characterization of the actuator mechanism to ensure the accuracy of the applied displacement. The linear stepper actuator uses a lead screw to convert the rotary motion of the rotor to linear motion. As specified on the motor datasheet, the stepper motor has a step angle of $1.8^{\circ}$ and the lead screw travels $50.8 \mu \mathrm{m}$ for one full step of the stepper motor. The integrated driver is capable of dividing the full $1.8^{\circ}$ step angle into 256 bits or "microsteps" by modulating the current input to the electromagnets in the stator, resulting in a gain value of $0.198 \mu \mathrm{m} /$ microstep. The applied displacement is characterized by attaching a scale bar onto the movable grip and measuring the actual displacement generated by the motor by taking images of the scale bar under a microscope and implementing image processing. A linear relationship is observed between the measured displacement (in $\mu \mathrm{m}$ ) and the motor command (in number of microsteps) with a coefficient of determination of $\mathrm{R}^{2}=0.9999$, and a gain value of $0.196 \mu \mathrm{m} /$ microstep which is in close proximity of the value reported on the datasheet.

The load cell is also calibrated before experimentation. It has a load capacity of $5 \mathrm{~N}$ and the rated output value specified in the calibration certification is $2.1095 \mathrm{mV} / \mathrm{V}$ resulting in a sensitivity of $4.2189 \mathrm{mV} / \mathrm{N}$. To enhance the measurement resolution, the raw output signal of the load cell is amplified and filtered using a custom-built signal conditioning circuit (Fig. 2(b)). The signal conditioning circuit consists of two passive RC low-pass filters, an operational amplifier with a gain value of 106.50, and a unity gain Sallen-Key low-pass filter. The load cell is calibrated using precise weights ranging from $1 \mathrm{~g}$ to $20 \mathrm{~g}$. The output voltage of the signal conditioner circuit is measured and recorded for each different load. There is a highly linear relationship between the measured output voltage and applied load with a coefficient of determination value of $R^{2}=1.0000$, and a sensitivity of $0.4339 \mathrm{~V} / \mathrm{N}$. The sensitivity value obtained by multiplying the raw output sensitivity (calculated from the calibration certification) with the amplifier gain of the signal conditioner circuit is in agreement with the calibration results. 


\section{Fluorescent microscopy imaging}

After the stretcher is assembled and calibrations are carried out, the stretcher is integrated with Olympus IX81 inverted microscope (Fig. 3(a) (Multimedia view)). The images are recorded with an Andor Ixon3-897 reinforced by a quantitative digital camera technology called Electron Multiplying Charge Coupled Device (EMCCD). A 10× air objective with 0.30 numerical aperture is used for magnification. The integrated system enables recording both white light and fluorescence images during stretching.

The maximum tracking distance of any bead embedded in the sample across the frame is $819.2 \mu \mathrm{m}$ due to the small field of view of the $10 \times$ objective and pixel size of the camera. Moreover, the sample is fixed at one end and stretched from

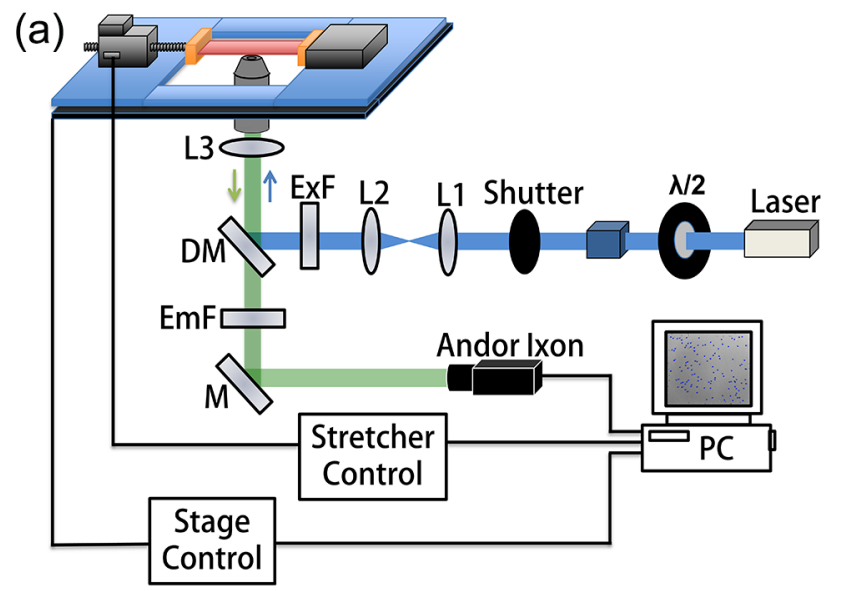

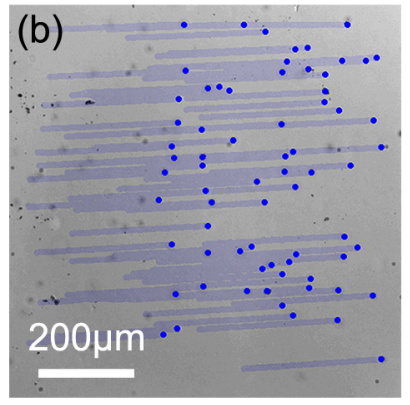

without stage control

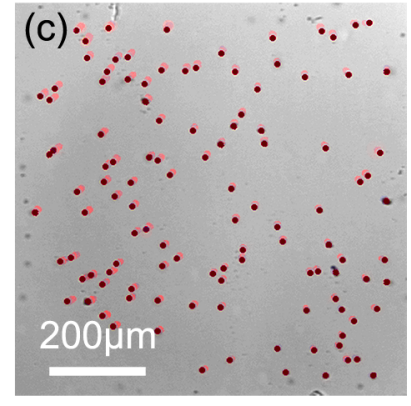

with stage control

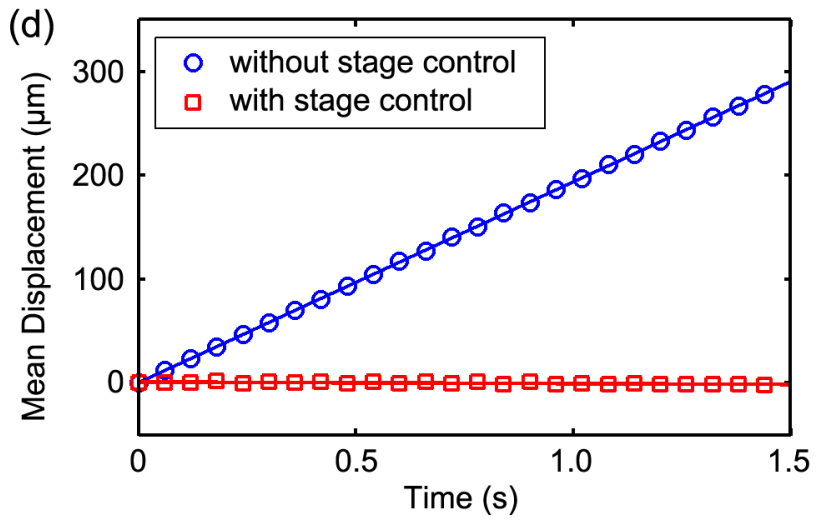

FIG. 3. (a) Schematic representation of optical setup. (b) Trajectories of beads recorded under white light illumination without stage control and (c) with stage control. (d) Comparison of the mean displacement of beads with and without stage control. (Multimedia view) [URL: http://dx.doi.org/ 10.1063/1.4941715.1] [URL: http://dx.doi.org/10.1063/1.4941715.2] the other end, as a result of which the group of fluorescent beads that are initially captured gradually move out of the field of view as the sample is stretched. This makes it challenging to track the same set of beads (and cells) at high strains as they move along the pulling direction and eventually leave the field of view. This is an issue commonly encountered in stretching systems and it can be resolved by implementing motion compensation, i.e., moving the entire setup to keep the objects of interest in the field of view. ${ }^{37}$ In the present work, this issue was addressed by using the motorized stage of the microscope to move the entire stretcher assembly in a direction opposite to the stretching direction to compensate for the motion of the sample. First, the center point of the sample is located by scanning the sample and recording the coordinates of its boundaries. Then, the stage is synchronized with the stretcher and the stage speed is adjusted to be half of the stretching speed. This way, the beads around the center point of the sample can be kept in the field of view while the sample is stretched up to high strains. A comparison of stretching without stage control and stretching with stage control to compensate for the motion of the beads is shown in Figs. 3(b) and 3(c) (Multimedia view), respectively. The same cross-head strain is applied to the samples in both cases and the trajectories of the beads are recorded. At 8\% cross-head strain, for the case of no stage control, 70 of the original 107 beads remain in the field of view (Fig. 3(b)) (Multimedia view), i.e., 37 beads move out of the field of view, whereas for the case of stretching with stage control, all 107 beads remain in the field of view (Fig. 3(c)) (Multimedia view). Corresponding mean displacements are given in Fig. 3(d) (Multimedia view).

\section{SAMPLE PREPARATION}

PDMS (Dow Corning Sylgard 184) is selected as the sample material in this study due to its low cost, easy fabrication, excellent optical transparency, chemical inertness, and non-toxicity properties, ${ }^{38,39}$ which make it a suitable substrate material for cell stretching studies. Precursor and curing agent are combined at a weight ratio of 10:1 in a weighing boat. Green-fluorescent polystyrene microspheres (Phosphorex, Inc.) of $5 \mu$ m diameter (Catalog Number: 2106B) are added to the mixture of pre-polymer and curing agent at a concentration of $3.2 \mu \mathrm{L} / \mathrm{g}$. This bead concentration provides an adequate number of beads in the field of view, as well as a measurable distance between the beads. The compound is spun with an average spin rate to ensure a homogeneous mixture.

Two different sample sets are prepared to demonstrate the capability of the present system to accurately measure local strain field. One set of samples has a rectangular geometry with a gage length of $30 \mathrm{~mm}$ and a width of $20 \mathrm{~mm}$. These samples are prepared by pouring the pre-polymer, curing agent, and fluorescent bead mixture into a Petri dish and cutting the desired rectangular shape after polymerization using a scalpel. The second set of samples has a dogbone shape with dimensions scaled by $1 / 3$ with respect to those specified by ASTM D412-C standard. The narrow neck section of dogbone samples has a length of $11 \mathrm{~mm}$ and a width of $2 \mathrm{~mm}$ and the overall gage length is $30 \mathrm{~mm}$. The dogbone samples are 


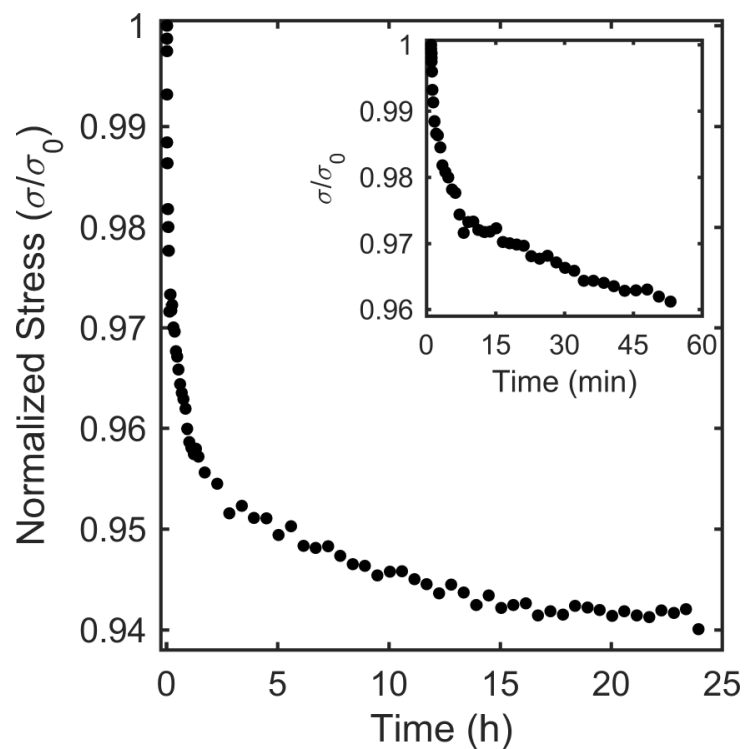

FIG. 4. Stress relaxation curve of PDMS at room temperature for $24 \mathrm{~h}$. The inset shows stress relaxation during the first hour.

prepared using an acrylic mold that is made using a $\mathrm{CNC}$ (Computer Numerical Control) laser cutter. Since all processes prior to pouring the mixture into the mold, especially mixing, generate air bubbles in the mixture, vacuum degassing is performed after mixture transferring to remove air bubbles. Once the mixture assumes its final shape in the mold, it is left to polymerize at room temperature for at least $48 \mathrm{~h}$.

Elastomers are known to exhibit stress relaxation under constant stretch, ${ }^{40}$ and it has also been shown that substrate stress relaxation affects cell spreading and proliferation. ${ }^{41}$ The stress relaxation response of the PDMS samples in this study is tested by keeping the samples under constant $40 \%$ stretch and recording the tension measured by the load cell. The nominal stress is then calculated as the ratio of tension to original crosssectional area, and all stress values are normalized by the initial stress. $6 \%$ stress relaxation is observed over a period of $24 \mathrm{~h}$ and the first $4 \%$ of this relaxation takes place during the first hour (Fig. 4). The capability to quantify this behavior through the integration of a load cell enables the present system to be used in future studies to investigate the effects of stress relaxation on adherent cells.

\section{RESULTS AND DISCUSSION}

\section{A. Tracking fluorescent microspheres}

In the present system, to compute local strain in a time-resolved manner, trajectories of embedded beads are determined from sequences of fluorescent microscopy images using a single-particle tracking (SPT) method. SPT is a commonly employed and useful technique in image processing for quantitative analysis of time-resolved microscopy data. $^{42-46}$ In addition to the ability to compute local strain and simultaneously image adherent cells, this method also has the advantage of performing strain measurement without making physical contact with the sample. This is a nontrivial advantage since non-contact measurement methods are more reliable for experiments on soft materials compared to contact measurement methods. ${ }^{47}$

Notably, an alternative non-contact method to experimentally quantify strain field is digital image correlation (DIC). DIC is an optical method where a speckle pattern is applied on the surface of the sample (usually by spray paint), and images of the speckle pattern before and after deformation are postprocessed to obtain 2D strain field. ${ }^{48}$ DIC has been successfully utilized to characterize strain field and Poisson's ratio for various materials, ${ }^{49-51}$ and to calibrate some of the previously reported cell stretching systems. ${ }^{12,13}$ However, it does suffer from a few shortcomings. While numerous commercial software packages are available, their operation is usually based on comparing two images, one before and one after deformation. Applying this step repeatedly to consecutive frames to compute time-resolved strain can be computationally intensive. Moreover, the speckle size distribution, subset size selection, data smoothing, and interpolation have been shown to influence measurement accuracy. ${ }^{52,53}$

In this work, SPT is selected over DIC mostly due to the physical nature of the experimental setup. The fluorescent beads embedded in the sample constitute a set of distinct markers of fixed size in the image plane rather than a random speckle pattern. Tracking the positions of these beads during stretching is a direct and relatively less computationally intensive method of measuring displacements. In the present method, the spatial resolution in displacement and strain measurement depends on the size and distribution density of embedded beads. While $5 \mu \mathrm{m}$ diameter beads at a moderate distribution density are used in this study for proof-of-concept purposes, it is possible to obtain very high spatial resolution using beads with diameters in the sub-micrometer range which are commercially available and have been used in the past to measure traction forces. ${ }^{27,54,55}$

To track the positions of embedded beads, videos of the PDMS samples are recorded at 16 frames/s by Andor Ixon EMCCD camera while the samples are stretched. Post-processing analysis on the images is carried out by a custom script written in MATLAB. Some features of IDL single-particle tracking code are adopted for localizing beads and linking the trajectories ${ }^{56,57}$ Pixel noise from the camera is filtered by a low pass filter and all images are averaged by a Gaussian function to generate smooth images.

Trajectories of the beads are then extracted from filtered images by three steps: identification of beads, determination of Cartesian coordinates, and sorting the locations to link the trajectory. The coordinates of beads are determined by computing the local maxima points in each image. Beads having more than one local maximum or beads that leave the field of view during the video recording are not taken into account. The trajectory of a bead is computed by estimating its position in consecutive frames. This is done by comparing each registered location in a pair of consecutive frames and minimizing the square displacement of centroids. Trajectories of beads are overlaid to the brightfield and fluorescence image (Figs. 5(a) and 5(b) (Multimedia view)) for low strains with no stage control to better illustrate the trajectories. The beads are excited at $485 \mathrm{~nm}$ and imaged by using $504 \mathrm{~nm}$ beam splitter and 521/15 $\mathrm{nm}$ emission filter. 

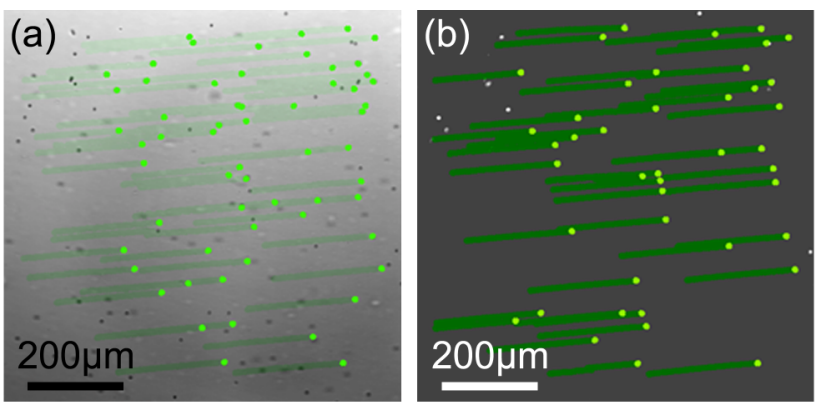

(c)

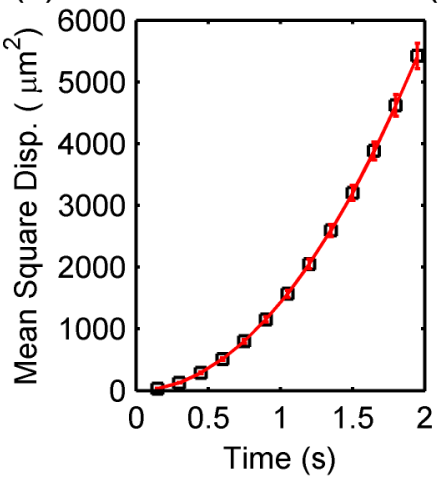

(d)

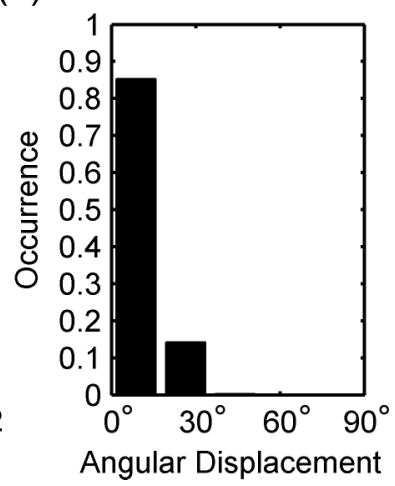

FIG. 5. Tracking the movement of single beads during the stretching and analysis of the motion. (a) Trajectory of beads recorded under white light and (b) fluorescence illumination captured at 16 frames/s for 2 s. After trajectories of beads from both illuminations were separately determined, they were overlaid as faded lines to the images. (c) The measured mean square displacement of the beads as a function of time at short time intervals was computed from trajectory of beads as well as (d) the angular displacement of beads along the direction of stretching. (Multimedia view) [URL: http://dx. doi.org/10.1063/1.4941715.3] [URL: http://dx.doi.org/10.1063/1.4941715.4]

The motility parameters can also be extracted from the bead trajectories, i.e., the mean square displacement (MSD) that is an indicator of the spread of trajectory (Fig. 5(c) (Multimedia view)). Since bead motion is driven by active directional stretching, MSD follows a parabolic trend that results from the migration pattern of the beads. Small errors on MSD even at longer lag times result from the uniform stretching of the beads and indicate that the beads do not move randomly. Finally, the angular displacements of the beads are measured and shown in Fig. 5(d) (Multimedia view). This is the angle that the trajectory curve makes with the horizontal axis. Probability close to $0^{\circ}$ has the highest magnitude indicating that the majority of the beads move horizontally during stretching. The smaller probability between $15^{\circ}$ and $30^{\circ}$ is due to the small lateral motion of the beads which is also apparent in Fig. 5(a) (Multimedia view). The highly directional and uniform motion of the beads indicates a good adhesion of the beads to the PDMS substrate and validates the assumption that the motion of the beads is a result of the applied uniaxial deformation. The results obtained from tracking the positions of fluorescent beads are then used for the evaluation of displacement and strain fields.

\section{B. Computation of local in-plane (2D) strain field}

Fluorescent microscopy and subsequent image processing provide the positions of embedded fluorescent beads as a function of time as the sample deforms. Treating the center points of the embedded fluorescent beads as material points, the motion of the deforming sample is described in Lagrangian coordinates by

$$
\boldsymbol{x}_{i}=\boldsymbol{\phi}\left(\boldsymbol{X}_{i}, t\right),
$$

where $\boldsymbol{X}_{i}$ denotes the initial positions of the points, and as the sample deforms, the instantaneous positions $\boldsymbol{x}_{i}$ of the same points at time $t$ are given by the function $\phi$. Once the positions of the fluorescent beads in consecutive frames are obtained from microscopy and image processing, the displacement vector is computed as

$$
\boldsymbol{u}_{i}\left(\boldsymbol{X}_{i}, t\right)=\boldsymbol{\phi}\left(\boldsymbol{X}_{i}, t\right)-\boldsymbol{X}_{i} .
$$

Positions (Eq. (1)) and displacements (Eq. (2)) of beads are quantities that are directly measured by microscopy. Strain, however, can be defined in terms of displacement in a number of ways. Therefore, the value of strain depends on the particular strain definition that one chooses. The appropriate choice of strain definition depends on the nature of deformation (elastic, inelastic, viscoelastic, etc.) and whether the deformation is small (infinitesimal) or large (finite). The choice of strain definition also becomes important when relating strain to stress, or when defining material properties such as elastic modulus or Poisson's ratio. ${ }^{58}$ The Green-Saint-Venant strain definition, commonly referred to as Green strain, was implemented in this study since the uniaxial stretcher is used to stretch the PDMS samples up to large strains, and the Green strain is an appropriate definition to describe large elastic deformations. ${ }^{59,60}$

To calculate the strain tensor, first the displacement gradient tensor is defined as

$$
\nabla \boldsymbol{u}_{i j}=\frac{\partial \boldsymbol{u}_{i}}{\partial \boldsymbol{X}_{j}} .
$$

The deformation gradient tensor is then defined in terms of the displacement gradient tensor as

$$
\boldsymbol{F}_{i j}=\nabla \boldsymbol{u}_{i j}+\boldsymbol{\delta}_{i j}
$$

where $\boldsymbol{\delta}_{i j}$ is the Kronecker delta. Then, the right Cauchy-Green deformation tensor is defined as

$$
\boldsymbol{C}_{i j}=\boldsymbol{F}_{k i} \boldsymbol{F}_{k j} \text {. }
$$

In formulating the strain tensor, the right Cauchy-Green deformation tensor is used instead of the deformation gradient tensor to ensure that the computed strain is decoupled from rigid body rotation. Finally, the Green-Saint-Venant strain tensor is defined as

$$
\boldsymbol{E}_{i j}=\frac{1}{2}\left(\boldsymbol{C}_{i j}-\boldsymbol{\delta}_{i j}\right)
$$

In 2D Euclidian space, with the $x$ and $y$ axes corresponding to the longitudinal (stretching direction) and transverse axes of the sample, the strain tensor in Eq. (6) can be expressed in component form as

$$
\boldsymbol{E}=\left[\begin{array}{ll}
\varepsilon_{x x} & \varepsilon_{x y} \\
\varepsilon_{y x} & \varepsilon_{y y}
\end{array}\right]=\left[\begin{array}{cc}
\varepsilon_{x x} & \gamma_{x y} / 2 \\
\gamma_{y x} / 2 & \varepsilon_{y y}
\end{array}\right],
$$

where the scalar components $\varepsilon_{x x}, \varepsilon_{y y}$, and $\gamma_{x y}$ which is equal to $\gamma_{y x}$ are the longitudinal normal, transverse normal, and the 
shear components of strain, respectively. To ensure consistency in comparing local strain computed from bead displacements with applied cross-head strain, the cross-head strain is also computed using the Green strain definition ${ }^{58}$ as

$$
\varepsilon_{C H}=\frac{1}{2}\left[\left(\frac{l}{l_{0}}\right)^{2}-1\right],
$$

where $\varepsilon_{C H}$ is the applied cross-head strain, $l_{0}$ is the original length of the sample, and $l$ is the stretched length calculated as the sum of the original length and the cross-head displacement applied by the linear actuator.

Using bead displacements, the local in-plane strain components are computed for a rectangular PDMS sample stretched with a cross-head speed of $200 \mu \mathrm{m} / \mathrm{s}$ up to 1.4 times its original length, corresponding to a cross-head strain of $48 \%$ using Eq. (8). Time evolutions of local strain components are shown in Fig. 6. The magnitude of the local longitudinal strain $\left(\varepsilon_{x x}\right)$ increases with time following a slightly nonlinear curve due to the quadratic terms in the Green strain definition. Since the sample is not constrained in the transverse direction in uniaxial loading, there is a negative transverse strain $\left(\varepsilon_{y y}\right)$ whose absolute magnitude also increases with time following a nonlinear curve. The magnitude of local shear strain $\left(\gamma_{x y}=\gamma_{y x}\right)$ remains approximately zero as expected in uniaxial loading.

Next, the local longitudinal strain computed from bead displacements is compared with the applied cross-head strain for rectangular and dogbone samples. Additionally, FEM simulations for the two sample geometries are carried out using a commercial software package to predict the distribution of strain over the entire sample geometry as well as to verify the value of local strain experimentally obtained at the center of the sample. The dimensions of the tested samples are used to construct the geometries in FEM with $x, y$, and $z$ axes of the Cartesian coordinate frame corresponding to the stretching direction, transverse direction, and out-of-plane direction, respectively. Once the geometries are constructed,

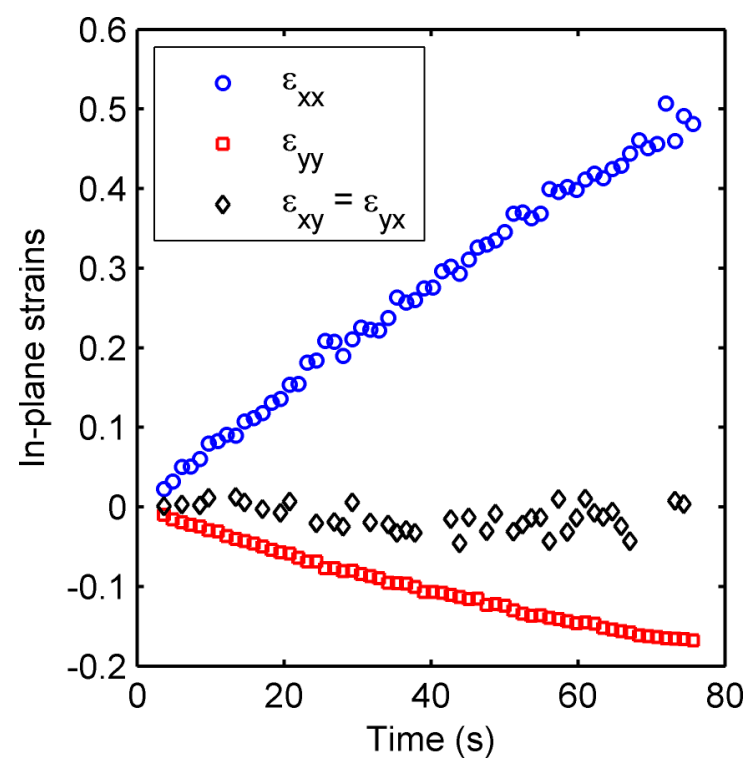

FIG. 6. Time evolution of local in plane strain components for a rectangular sample.
TABLE I. Values of nodal parameters used in FEM.

\begin{tabular}{lcc}
\hline \hline Sample geometry & Number of elements & Number of degrees of freedom \\
\hline Rectangular & 14270 & 92352 \\
Dogbone & 21730 & 115326 \\
\hline \hline
\end{tabular}

a tetrahedral mesh is applied. Table I lists the number of mesh elements and number of degrees of freedom used in the simulation for each sample geometry. Regarding boundary conditions, one end of the sample is fixed by prescribing zero displacements in $x, y$, and $z$ directions on all the nodes at this end, while uniaxial stretching is simulated by applying displacement in $x$ direction and prescribing zero displacement in $y$ and $z$ directions on all the nodes at the opposite end. The value of the applied displacement is adjusted such that the sample is stretched up to 1.4 times its original length. As
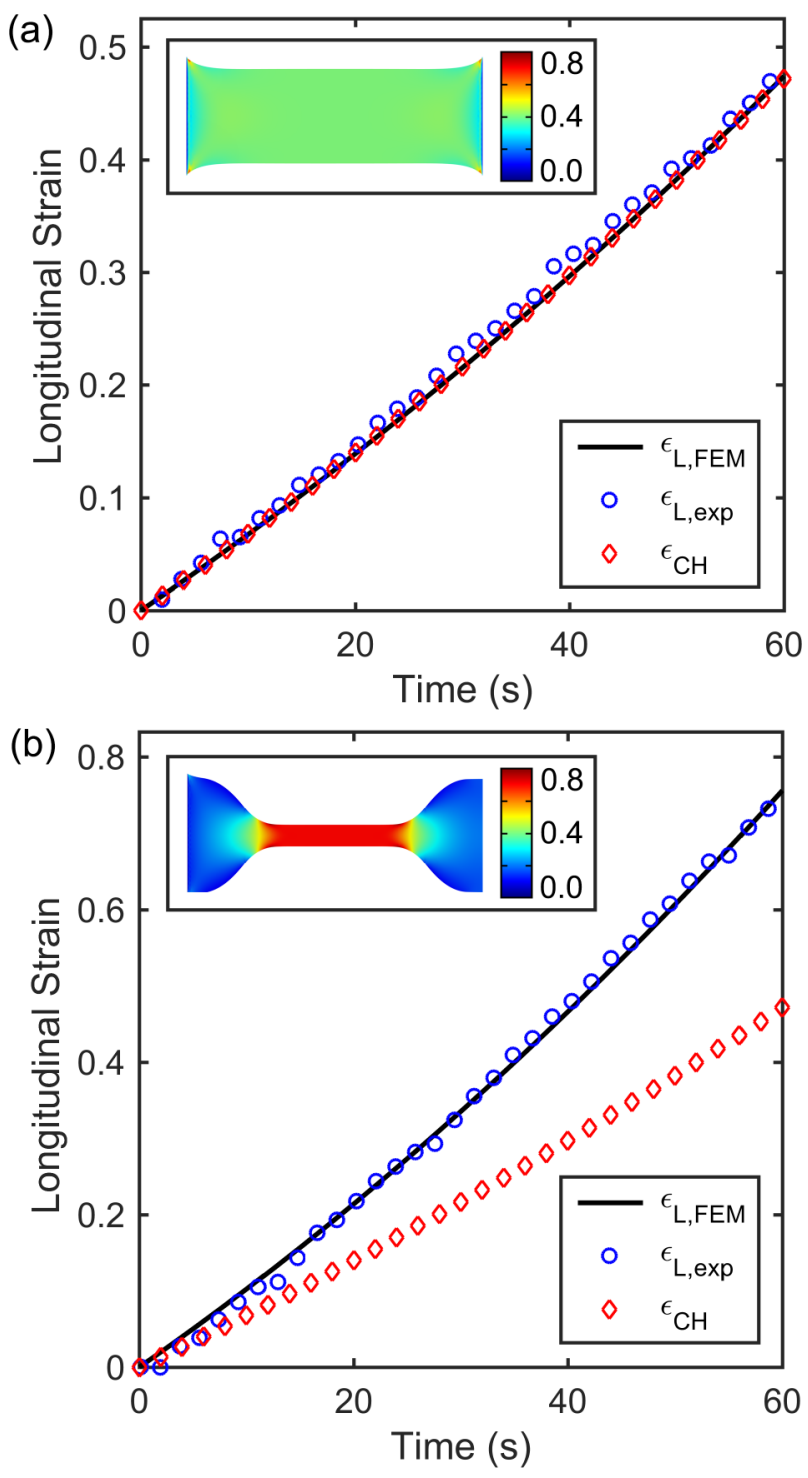

FIG. 7. Comparison of experimentally measured local longitudinal strain $\left(\varepsilon_{\mathrm{L}, \text { exp }}\right)$, local strain obtained from FEM $\left(\varepsilon_{\mathrm{L}, \mathrm{FEM}}\right)$, and cross-head strain $\left(\varepsilon_{\mathrm{CH}}\right)$ for a rectangular sample (a) and for a dogbone sample (b). Top left insets in each panel show the FEM results illustrating the distribution of longitudinal strain over the entire sample geometry. 
the material model, the first-order Mooney-Rivlin constitutive relation is used. This constitutive relation was developed to model the hyperelastic behavior of rubber-like materials under large deformations. ${ }^{61,62}$ The model requires two material constants, namely $\mathrm{c}_{10}$ and $\mathrm{c}_{01}$, whose values are taken as $90.35 \mathrm{kPa}$ and $12.82 \mathrm{kPa}$, respectively. These values have previously been reported to model PDMS within the range of strain used in this work. $^{13}$

Fig. 7(a) shows the comparison of local and cross-head strain for a rectangular sample. Since strain is distributed uniformly over the sample, as depicted by the color-plot obtained from FEM (top left inset in Fig. 7(a)), the local longitudinal strain and applied cross-head strain overlap. Furthermore, the experimentally obtained values of local strain at the center of the sample match well with the local strain given by FEM. The same comparison for the dogbone sample geometry is shown in Fig. 7(b). The spatial distribution of strain depicted by FEM results (top left inset in Fig. 7(b)) shows that most of the deformation is confined to the narrow neck region, while wider end sections undergo much less deformation. As a result, the local strain at the center is larger than the cross-head strain since the latter is an average value. A quantitative comparison shows that for the particular dogbone sample geometry used in this study, the experimentally obtained value of local strain at the center is $77 \%$ for an applied cross-head strain of $48 \%$. This strain distribution pattern is a characteristic of dogbone samples in uniaxial tension, where the local strain at the center is greater than the cross-head strain, and the difference between the two increases as deformation becomes larger. ${ }^{36}$ The local strain computed from bead displacement at the center of the dogbone sample also matches well with the local strain values obtained from FEM analysis (Fig. 7(b)), illustrating the capability of the present system to accurately measure local strain in samples with non-uniform strain distribution.

\section{Demonstration of cell stretching}

A unibody cell-stretching sample is prepared from PDMS, having gripping regions at both ends and a well in between that contains adherent cells and culture media (Fig. 8(a)). The sample is prepared by pouring PDMS mixture into a plexiglas mold and curing it as described above. A singlepiece flexible substrate prevents any fluid flowing out during stretching. If the sample is prepared by bonding two pieces of PDMS, loss of media can be observed during stretching because the piece making up the well walls tends to detach at high strains. To enhance biocompatibility, the hydrophobic surface of PDMS is treated with concentrated $\mathrm{HCl}$ for $3 \mathrm{~h}$, and washed several times with Phosphate Buffered Saline (PBS) buffer until it is neutralized, and cleaned for surface coating. Rat-tail collagen (Olaf Pharmaceuticals) from stock is applied to the surface to enhance cell adhesion, and the sample is incubated at $37^{\circ} \mathrm{C}$ until a monolayer of fibers forms on the surface. HeLa S3 cells are cultured in a cell culture dish until they reach $80 \%$ confluency. They are then transferred into the collagen-coated PDMS well and incubated overnight until cells reach $60 \%$ confluency to ensure that the functionalized PDMS surface is a suitable substrate for cell attachment and growth.

Samples with adherent cells are then stretched and displacements of beads are computed by tracking their positions as discussed in Secs. IV A and IV B (Fig. 8(b)). Brightfield and fluorescent microscopy images are analyzed to localize the beads with respect to the cells since both beads and cells can be observed in the brightfield image
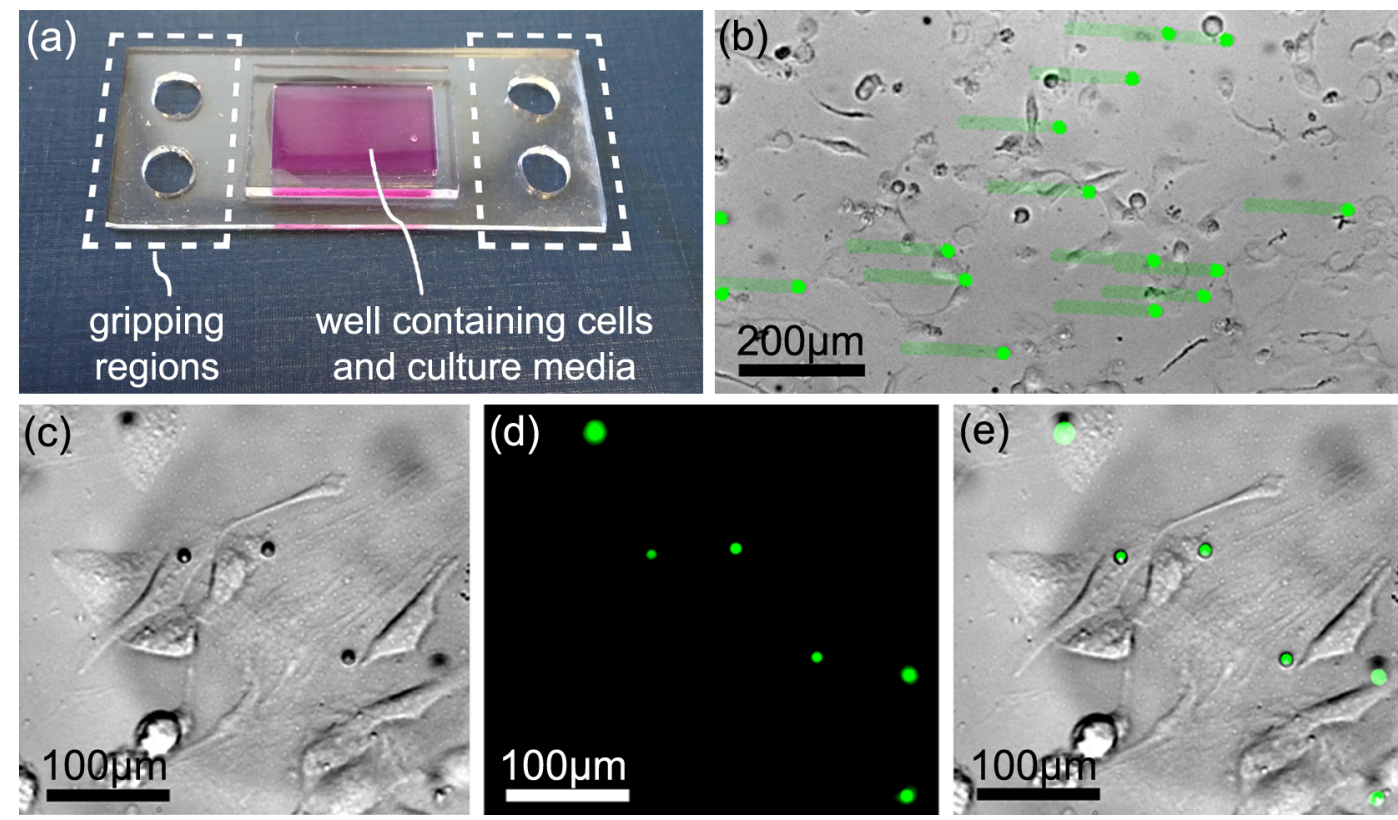

FIG. 8. Preparation of PDMS samples for cell-stretching and tracking of fluorescent beads around cells. (a) $24 \times 55$ mm unibody PDMS sample prepared for cell attachment and stretching, having a $12 \times 19 \mathrm{~mm}$ well that contains culture media and is coated with collagen to enhance cell adhesion. Unibody structure with the well prevents leakage or outflow of culture media during stretching up to high strains. (b) Trajectories of fluorescent beads are overlaid onto the brightfield image of adherent cells for a sample that is stretched without motion compensation to better illustrate the bead trajectories. ((c)-(e)) Close-up brightfield, fluorescence, and overlaid images showing beads embedded in the sample with adherent cells. 
(Fig. 8(c)), whereas the fluorescent image (Fig. 8(d)) shows the beads only. Fluorescence emission intensity of beads is high offering a suitable system for labeling and strain computation. The overlay of brightfield and fluorescent signals clearly demonstrates that beads are localized around the cells (Fig. 8(e)). The brightness of beads remains unchanged during preparation and surface coating of PDMS samples, providing a strong signal to track during stretching.

\section{CONCLUSIONS AND OUTLOOK}

A uniaxial cell stretcher is developed by integrating a custom-built tensile stretcher platform with fluorescent microscopy for the measurement of local in-plane strain components and simultaneous imaging of biological cells. PDMS samples with embedded fluorescent beads are used to demonstrate particle tracking and subsequent displacement and strain measurement techniques. The time evolution of local longitudinal, transverse, and shear components of strain is computed for a PDMS sample of rectangular geometry up to $48 \%$ longitudinal strain. The Green strain definition is used throughout the entire study since it is an appropriate formulation for large deformations. The magnitudes of normal strain components are found to increase nonlinearly with time due to the quadratic terms in the Green strain definition while shear strain remains close to zero, as expected in uniaxial loading. Local longitudinal strain values are compared with the applied cross-head strain for two different sample geometries. For the rectangular samples, the local strain at the center overlaps with the average cross-head strain, indicating uniform strain distribution. For the dogbone samples, the local longitudinal strain at the center is measured to be $77 \%$ while the applied cross-head strain is $48 \%$. This difference is caused by the non-uniform distribution of strain as predicted by FEM analysis. These results emphasize the fact that significant errors may arise when cross-head strain measures are used in cell stretching experiments with sample geometries of non-uniform cross section and demonstrate the value of the present technique's capability to accurately measure local strain.

Compatibility of the present uniaxial stretching platform with biological samples is also demonstrated. Unibody PDMS samples having a small well to contain cells and culture media are prepared using a plexiglas mold. Sample surface is coated with collagen to promote cell adhesion, and HeLa S3 cells are plated on the samples. Cells reach $60 \%$ confluency when incubated overnight, demonstrating the suitability of the samples for cell attachment and proliferation. Finally, samples with adherent cells are stretched and images of cells and embedded fluorescent beads are recorded simultaneously. Beads can be detected separately and tracked using fluorescent microscopy. Localization of beads with respect to adherent cells is also demonstrated by overlaying fluorescent images of beads onto brightfield images of cells.

The instrumentation and technique developed in the present work provide a robust platform for future mechanobiology studies with the ability to perform cell stretching and accurately quantify relevant local substrate deformation.

\section{ACKNOWLEDGMENTS}

This work is financially supported by the Scientific and Technological Research Council of Turkey (TUBITAK) under Grant Nos. 112E580 and 112T823. O.B.A. is supported by 2210 national scholarship predoctoral training program. We are grateful to Olaf Pharmaceuticals for providing purified type I collagen.

${ }^{1}$ D. E. Ingber, FASEB J. 20, 811 (2006)

${ }^{2}$ T. D. Brown, J. Biomech. 33, 3 (2000).

${ }^{3}$ T. Takemasa, K. Sugimoto, and K. Yamashita, Exp. Cell Res. 230, 407 (1997).

${ }^{4}$ P. C. Dartsch and E. Betz, Basic Res. Cardiol. 84, 268 (1989).

${ }^{5}$ V. P. Shirinsky, A. S. Antonov, K. G. Birukov, A. V. Sobolevsky, Y. A. Romanov, N. V. Kabaeva, G. N. Antonova, and V. N. Smirnov, J. Cell Biol. 109, 331 (1989).

${ }^{6}$ K. Naruse, T. Yamada, and M. Sokabe, Am. J. Physiol. 274, 1532 (1998).

${ }^{7}$ K. Hayakawa, A. Hosokawa, K. Yabusaki, and T. Obinata, Zool. Sci. 17, 617 (2000).

${ }^{8}$ K. Hayakawa, N. Sato, and T. Obinata, Exp. Cell Res. 268, 104 (2001).

${ }^{9}$ M. J. Yost, D. Simpson, K. Wrona, S. Ridley, H. J. Ploehn, T. K. Borg, and L. Terracio, Am. J. Physiol-Heart C 279, 3124 (2000).

${ }^{10}$ S. Jungbauer, H. Gao, J. P. Spatz, and R. Kemkemer, Biophys. J. 95, 3470 (2008).

${ }^{11}$ J. L. Balestrini, J. K. Skorinko, A. Hera, G. R. Gaudette, and K. L. Billiar, Biomech. Model. Mechanobiol. 9, 329 (2010).

${ }^{12}$ Y. Morita, S. Watanabe, Y. Ju, and S. Yamamoto, Cell Biochem. Biophys. 67, 1249 (2013).

${ }^{13}$ W. W. Ahmed, M. H. Kural, and T. A. Saif, Acta Biomater. 6, 2979 (2010).

${ }^{14}$ C.-F. Lee, C. Haase, S. Deguchi, and R. Kaunas, Biochem. Biophys. Res. Commun. 401, 344 (2010).

${ }^{15}$ Y. Shao, X. Tan, R. Novitski, M. Muqaddam, P. List, L. Williamson, J. Fu, and A. P. Liu, Rev. Sci. Instrum. 84, 114304 (2013).

${ }^{16}$ A. M. Goldyn, B. A. Rioja, J. P. Spatz, C. Ballestrem, and R. Kemkemer, J. Cell Sci. 122, 3644 (2009).

${ }^{17}$ A. Carisey, R. Tsang, A. M. Greiner, N. Nijenhuis, N. Heath, A. Nazgiewicz, R. Kemkemer, B. Derby, J. Spatz, and C. Ballestrem, Curr. Biol. 23, 271 (2013).

${ }^{18}$ Y. Chen, A. M. Pasapera, A. P. Koretsky, and C. M. Waterman, Proc. Natl. Acad. Sci. U. S. A. 110, 2352 (2013).

${ }^{19}$ A. J. Banes, J. Gilbert, D. Taylor, and O. Monbureau, J. Cell Sci. 75, 35 (1985).

${ }^{20}$ S. Hasegawa, S. Sato, S. Saito, Y. Suzuki, and D. M. Brunette, Calcif. Tissue Int. 37, 431 (1985).

${ }^{21}$ F. Shi, Y.-J. Chiu, Y. Cho, T. A. Bullard, M. Sokabe, and K. Fujiwara, Cardiovasc. Res. 73, 813 (2007).

${ }^{22}$ A. Eekhoff, N. Bonakdar, J. L. Alonso, B. Hoffmann, and W. H. Goldmann, Biochem. Biophys. Res. Commun. 406, 229 (2011).

${ }^{23}$ C. P. Ursekar, S.-K. Teo, H. Hirata, I. Harada, K.-H. Chiam, and Y. Sawada, PLoS One 9, e90665 (2014).

${ }^{24}$ S. Kippenberger, A. Bernd, S. Loitsch, M. Guschel, J. Muller, J. BereiterHahn, and R. Kaufmann, J. Invest. Dermatol. 114, 408 (2000).

${ }^{25}$ N. Gavara, P. Roca-Cusachs, R. Sunyer, R. Farre, and D. Navajas, Biophys. J. 95, 464 (2008).

${ }^{26}$ C. M. Waters, M. R. Glucksberg, E. P. Lautenschlager, C.-W. Lee, R. M. V. Matre, R. J. Warp, U. Savla, K. E. Healy, B. Moran, D. G. Castner, and J. P. Bearinger, J. Appl. Physiol. 91, 1600 (2001).

${ }^{27}$ T. Dey, M. C. Mann, and W. H. Goldmann, Biochem. Biophys. Res. Commun. 413, 541 (2011).

${ }^{28}$ N. Bonakdar, J. Luczak, L. Lautscham, M. Czonstke, T. M. Koch, A. Mainka, T. Jungbauer, W. H. Goldmann, R. Schröder, and B. Fabry, Biochem. Biophys. Res. Commun. 419, 703 (2012).

${ }^{29}$ G. P. Raeber, M. P. Lutolf, and J. A. Hubbell, Biomech. Model. Mechanobiol. 7, 215 (2008)

${ }^{30}$ W. W. Ahmed, T. Wolfram, A. M. Goldyn, K. Bruellhoff, B. A. Rioja, M. Moller, J. P. Spatz, T. A. Saif, J. Groll, and R. Kemkemer, Biomaterials 31, 250 (2010).

${ }^{31}$ J. D. King, D. Hayes, K. Shah, S. L. York, P. Sethu, and M. M. Saunders, in Mechanics of Biological Systems and Materials, edited by F. Barthelat, C. Korach, P. Zavattieri, B. C. Prorok, and K. J. Grande-Allen (Springer International Publishing, 2015), Vol. 7, pp. 61-67. 
${ }^{32}$ J. A. Gilbert, P. S. Weinhold, A. J. Banes, G. W. Link, and G. L. Jones, J. Biomech. 27, 1169 (1994).

${ }^{33}$ G. P. Raeber, J. Mayer, and J. A. Hubbell, Biomech. Model. Mechanobiol. 7, 203 (2008).

${ }^{34}$ D. Wang, Y. Xie, B. Yuan, J. Xu, P. Gong, and X. Jiang, Integr. Biol. 2, 288 (2010).

${ }^{35}$ J. Imsirovic, T. J. Wellman, J. R. Mondonedo, E. Bartolak-Suki, and B. Suki, PLoS One 10, e0140283 (2015).

${ }^{36} \mathrm{~F}$. Schneider, T. Fellner, J. Wilde, and U. Wallrabe, J. Micromech. Microeng. 18, 065008 (2008).

${ }^{37}$ A. Gerstmair, G. Fois, S. Innerbichler, P. Dietl, and E. Felder, J. Appl. Physiol. 107, 613 (2009)

${ }^{38}$ G. M. Whitesides, Nature 442, 368 (2006).

${ }^{39}$ E. Sollier, C. Murray, P. Maoddi, and D. Di Carlo, Lab Chip 11, 3752 (2011)

${ }^{40} \mathrm{~K}$. Yamaguchi, A. G. Thomas, and J. J. C. Busfield, Int. J. Nonlinear Mech. 68, 66 (2015).

${ }^{41}$ O. Chaudhuri, L. Gu, M. Darnell, D. Klumpers, S. A. Bencherif, J. C. Weaver, N. Huebsch, and D. J. Mooney, Nat. Commun. 6, 6365 (2015).

${ }^{42}$ S. S. Rogers, T. A. Waigh, X. Zhao, and J. R. Lu, Phys. Biol. 4, 220 (2007)

${ }^{43}$ M. K. Cheezum, W. F. Walker, and W. H. Guilford, Biophys. J. 81, 2378 (2001).

${ }^{44}$ M. J. Saxton, Nat. Methods 5, 671 (2008).

${ }^{45}$ E. Meijering, O. Dzyubachyk, and I. Smal, Methods Enzymol. 504, 183 (2012).

${ }^{46}$ N. Chenouard, I. Smal, F. d. Chaumont, M. Maska, I. F. Sbalzarini, Y Gong, J. Cardinale, C. Carthel, S. Coraluppi, M. Winter, A. R. Cohen, W. J. Godinez, K. Rohr, Y. Kalaidzidis, L. Liang, J. Duncan, H. Shen, Y. Xu,
K. E. G. Magnusson, J. Jalden, H. M. Blau, P. Paul-Gilloteaux, P. Roudot, C. Kervrann, F. Waharte, J.-Y. Tinevez, S. L. Shorte, J. Willemse, K. Celler, G. P. v. Wezel, H.-W. Dan, Y.-S. Tsai, C. O. d. Solorzano, J.-C. Olivo-Marin, and E. Meijering, Nat. Methods 11, 281 (2014).

${ }^{47}$ A. J. W. McClung, G. P. Tandon, K. E. Goecke, and J. W. Baur, Polym. Test. 30, 140 (2011).

${ }^{48}$ B. Pan, K. Qian, H. Xie, and A. Asundi, Meas. Sci. Technol. 20, 062001 (2009).

${ }^{49}$ J. Zhang, Y. Cai, W. Ye, and T. X. Yu, Opt. Laser Eng. 49, 200 (2011).

${ }^{50} \mathrm{D}$. Tscharnuter, M. Jerabek, Z. Major, and R. W. Lang, Mech. Time-Depend. Mater. 15, 15 (2011).

${ }^{51}$ R. H. Pritchard, P. Lava, D. Debruyne, and E. M. Terentjev, Soft Matter 9 , 6037 (2013)

${ }^{52}$ D. Lecompte, A. Smits, S. Bossuyt, H. Sol, J. Vantomme, D. V. Hemelrijck, and A. M. Habraken, Opt. Laser Eng. 44, 1132 (2006).

${ }^{53}$ P. Lava, S. Cooreman, and D. Debruyne, Opt. Laser Eng. 48, 457 (2010).

${ }^{54}$ J. P. Butler, I. M. Tolic-Norrelykke, B. Fabry, and J. J. Fredberg, Am. J. Physiol.: Cell Physiol 282, 595 (2002).

${ }^{55}$ S. G. Knoll, M. Y. Ali, and M. T. A. Saif, J. Vis. Exp. 91, e51873 (2014)

${ }^{56}$ J. C. Crocker and D. G. Grier, J. Colloid Interface Sci. 179, 298 (1996).

${ }^{57}$ D. Blair and E. Dufresne, The Matlab Particle Tracking Code Repository, http://site.physics.georgetown.edu/matlab/.

${ }^{58}$ O. Starkova and A. Aniskevich, Polym. Test. 29, 310 (2010).

${ }^{59}$ J. Lubliner, Plasticity Theory (Dover Publications, New York, 2008).

${ }^{60}$ A. I. Lurie, Theory of Elasticity (Springer, Berlin, 2005).

${ }^{61}$ M. Mooney, J. Appl. Physiol. 11, 582 (1940).

${ }^{62}$ R. S. Rivlin and D. W. Saunders, Philos. Trans. R. Soc., A 243, 251 (1951). 